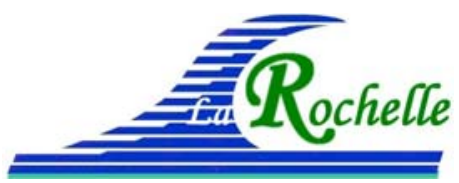

XVèmes Journées Nationales Génie Côtier - Génie Civil

La Rochelle, 29 au 31 mai 2018

DOI:10.5150/jngcgc.2018.046

(c) Editions Paralia CFL

disponible en ligne - http://www.paralia.fr - available online

\title{
L'extension en mer de l'Anse du Portier à Monaco
}

\section{Pierre ARISTAGHES ${ }^{\text {, }}$, Valérie BLANCHET ${ }^{2}$, David COMPTE ${ }^{\mathbf{1}}$, Arnaud GREVOZ ${ }^{1}$, Brice QUENOUILLE ${ }^{1}$, Maria TELES ${ }^{2}$, Antoine WEULERSSE ${ }^{2}$}

1. Bouygues TP, 1 avenue Freyssinet, 78280 - Guyancourt, France.

p.aristaghes@bouygues-construction.com

2. Egis Ports, 15 avenue du Centre, 78280 - Guyancourt, France.

valerie.blanchet@egis.fr

\section{Résumé :}

La conception de l'extension en mer de l'Anse du Portier à Monaco a nécessité une approche multidisciplinaire et souvent originale compte-tenu du caractère non courant du projet et du contexte lié au site (exposition aux houles, proximité de zones naturelles et portuaires sensibles, raccordement avec des ouvrages existants complexes, niveau de séisme associé à des ouvrages de grande hauteur sur des sols sensibles).

Parmi les problèmes à résoudre, on évoquera tout d'abord la recherche de niveaux de promenade aussi bas que possible du point de vue architectural, mais compatibles avec les franchissements de tempête, ainsi que la recherche de coefficients de réflexion minimaux pour ne pas risquer d'impacter l'agitation dans le port existant, ce qui a amené à concevoir des caissons à chambre de dissipation très efficaces en termes de limitation des franchissements et des réflexions.

La présence d'un nouveau port d'animation dont la position était figée au cœur de la nouvelle extension urbaine a nécessité des optimisations très poussées de dispositifs anti-franchissement, aussi intégrés dans le contexte urbain que possible (parapets en chicane, systèmes de drainage compatibles avec des zones piétonnières de qualité, "digue-piscine", ...).

La recherche d'écoconception a conduit à intégrer celle-ci dans les ouvrages d'infrastructure, et non pas comme des objets supplémentaires à côté des ouvrages, ce qui a nécessité des évaluations d'efforts de houle sur des configurations atypiques.

La proximité immédiate de fondations d'immeubles au droit des caissons de ceinture a également nécessité des approches originales pour la conception parasismique des soutènements.

Mots-clés : Génie côtier, Hydraulique maritime, Travaux maritimes, Environnement littoral, Ecosystèmes côtiers, Séisme, Digue, Monaco.

\section{Contexte général}

Le projet de l'Anse du Portier à Monaco consiste en une extension urbaine en mer de 6 ha, située entre Port Hercule et les plages du Larvotto (figure 1). Le plan-masse retenu 


\section{Thème 4 - Ouvrages portuaires, offshore et de plaisance}

inclut des zones résidentielles, des espaces publics, un port d'animation, ainsi qu'un "caisson-piscine" destiné à compléter la protection du port.

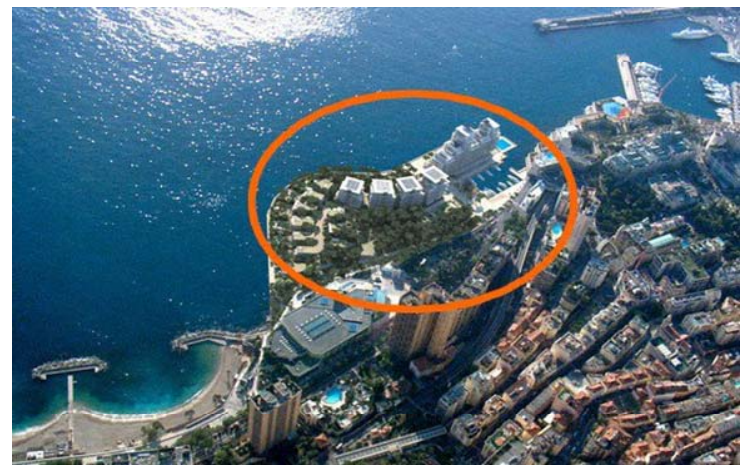

Figure 1. Implantation du projet.

Le présent article porte sur la conception de l'infrastructure marine, réalisée par le groupement Bouygues TP - Egis Ports, sous le contrôle technique de Socotec et Cowi, pour le compte de la SAM Anse du Portier et de l'Etat monégasque, lequel a choisi Setec pour AMO. Cette conception s'est faite en liaison étroite avec les architectes du projet (les cabinets Valode \& Pistre Architectes et Renzo-Piano Building Workshop, et Michel Desvigne Paysagiste).

\section{Conditions de site}

\section{$2.1 \underline{\text { Houles }}$}

Une étude complète de hindcasting a été confiée à Globocéan, qui a conduit à définir des séries temporelles d'états de mer en différents points proches du site (figure 2).

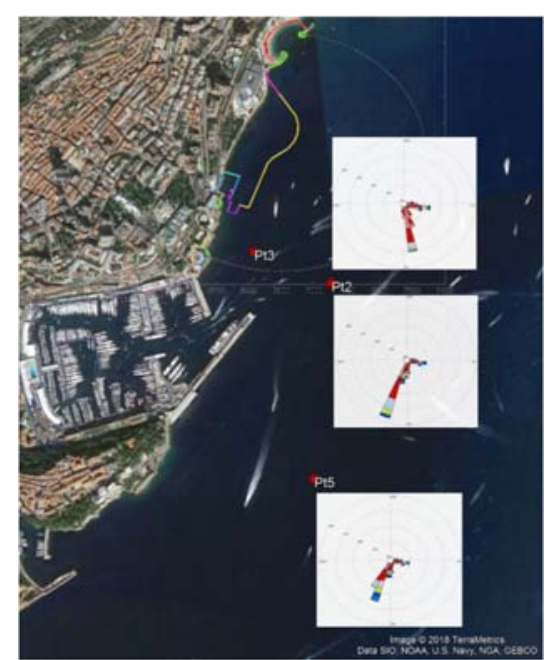

Figure 2. Points d'extraction à proximité du projet et roses des houles. 


\section{XVèmes Journées Nationales Génie Côtier - Génie Civil La Rochelle, 29 au 31 mai 2018}

Contrairement à ce qui se pratique souvent, le modèle océano-météorologique a été prolongé jusqu'aux abords immédiats du site, en des points situés loin de la zone de déferlement, plutôt que d'arrêter le hindcasting en un point dit "au large" et de propager ensuite ces états de mer depuis le large jusqu'à la côte avec des logiciels de type Swan.

Ceci permet de conserver toute l'information spectrale jusqu'au site, en évitant de devoir réduire un spectre directionnel à un état de mer "équivalent" caractérisé par un triplet $\left(H_{m 0}, T_{p}, \theta\right)$, opération qui entraîne inévitablement une perte d'information notamment dans le cas courant de superposition d'une houle et d'une mer de vent de directions distinctes. Par ailleurs, le vent et son action sur les vagues continuent à être représenté jusqu'au site.

A cela s'ajoute la nécessité pour l'étude du port d'animation de pouvoir prendre en compte l'effet de la digue flottante de Port Hercule sur les houles de secteur Sud. Des valeurs conservatives du coefficient de transmission $C_{t}$ (sur la base de résultats de mesures d'agitation en cuve à houle effectuées lors de la conception de Port Hercule) ont été injectées dans le modèle de propagation du hindcasting, afin d'obtenir une houle de Sud réaliste au point Pt3. De ces séries temporelles ont été tirées les valeurs extrêmes, dont les résultats pour le point $\mathrm{Pt} 2$ sont donnés au tableau 1 à titre d'exemple.

Tableau 1. Valeurs extrêmes des hauteurs de houle au point Pt2.

\begin{tabular}{|c|c|c|c|c|c|c|c|c|c|}
\hline \multirow[b]{2}{*}{$\begin{array}{l}\text { Point de } \\
\text { référence }\end{array}$} & \multirow[b]{2}{*}{$R$ (ans) } & \multicolumn{4}{|c|}{ Secteur SW } & \multicolumn{4}{|c|}{ Secteur NE } \\
\hline & & $\begin{array}{l}H_{m 0} \\
(m)\end{array}$ & $T_{p}(s)$ & $\begin{array}{c}\text { Direction } \\
\text { (\%/Nord) }\end{array}$ & $\begin{array}{c}90 \% \text { int. } \\
\text { conf. sur } \\
H_{m 0}\end{array}$ & $\begin{array}{l}H_{m 0} \\
(m)\end{array}$ & $T_{p}(s)$ & $\begin{array}{c}\text { Direction } \\
\text { (\%/Nord) }\end{array}$ & $\begin{array}{c}90 \% \text { int. } \\
\text { conf. sur } \\
H_{m 0}\end{array}$ \\
\hline \multirow{5}{*}{$\begin{array}{c}P t 2 \\
(\grave{a}-60 \mathrm{~m} \\
N G F)\end{array}$} & 1 & 2.3 & $7-11$ & $170-200$ & $2.2-2.4$ & 2.0 & $6-9$ & $100-130$ & $1.9-2.0$ \\
\hline & 5 & 2.8 & $8-11$ & $170-200$ & $2.7-3.0$ & 2.4 & $7-10$ & $100-130$ & $2.2-2.6$ \\
\hline & 10 & 3.0 & $9-11$ & $170-200$ & 2.8-3.2 & 2.7 & $7-10$ & $100-130$ & 2.4-2.9 \\
\hline & 50 & 3.5 & $9-11$ & $170-200$ & $3.2-3.8$ & 3.3 & $8-10$ & $100-120$ & $2.9-3.7$ \\
\hline & 100 & 3.7 & $9-11$ & $180-200$ & $3.4-4.0$ & 3.5 & $8-10$ & $100-120$ & $3.1-4.0$ \\
\hline
\end{tabular}

\subsection{Niveaux d'eau}

Les niveaux d'eau maximaux qui ont été considérés sont notés sur le tableau 2. Pour la conception de certains dispositifs anti-franchissement, notamment dans la zone du port d'animation, on a été amené à étudier la concomitance des valeurs extrêmes des hauteurs $H_{m 0}$ et des niveaux d'eau $Z_{w}$.

Tableau 2. Niveaux d'eau maximaux considérés.

\begin{tabular}{|c|c|c|c|c|}
\hline $\begin{array}{l}\text { Période de } \\
\text { retour }\end{array}$ & $\begin{array}{c}\text { Niveau de marée } \\
\text { astronomique } \\
\text { [m NGF] }\end{array}$ & $\begin{array}{l}\text { Valeur de } \\
\text { surcote } \\
\text { [m] }\end{array}$ & $\begin{array}{c}\text { Elévation du } \\
\text { niveau d'eau - } \\
\text { horizon } 2100[\mathrm{~m}]\end{array}$ & $\begin{array}{l}\text { Niveau d'eau } \\
\text { [m NGF] }\end{array}$ \\
\hline 1 an & +0.37 & +0.30 & +0.75 & +1.42 \\
\hline 10 ans & +0.37 & +0.49 & +0.75 & +1.61 \\
\hline 100 ans & +0.37 & +0.68 & +0.75 & +1.80 \\
\hline
\end{tabular}




\section{Thème 4 - Ouvrages portuaires, offshore et de plaisance}

Pour ce faire, on a appliqué la méthode DEFRA du British Department of Environment, Food and Rural Affairs, qui consiste à caractériser le degré de corrélation entre hauteur $H_{s}$ et niveau d'eau $Z_{w}$ maximaux sur un cycle de $12 \mathrm{~h}$ par un coefficient $C$ défini par :

$$
C=\frac{p\left(H_{s}>h \text { et } Z_{w}>z\right)}{p\left(H_{s}>h\right) \cdot p\left(Z_{w}>z\right)}
$$

L'analyse des séries temporelles de $H_{m o}$ en $\mathrm{Pt} 2$ et du marégraphe de Fontvieille sur la période 2000-2013 a conduit à une valeur de $C$ de l'ordre de 20 pour des échantillons de durée de retour voisine de 1 an, comme le montre les points rouges de la figure 3 , où la durée de retour $R$ est définie comme $1 /\left(730 \mathrm{p}\left(H_{s}>h\right.\right.$ et $\left.\left.Z_{w}>z\right)\right)$.

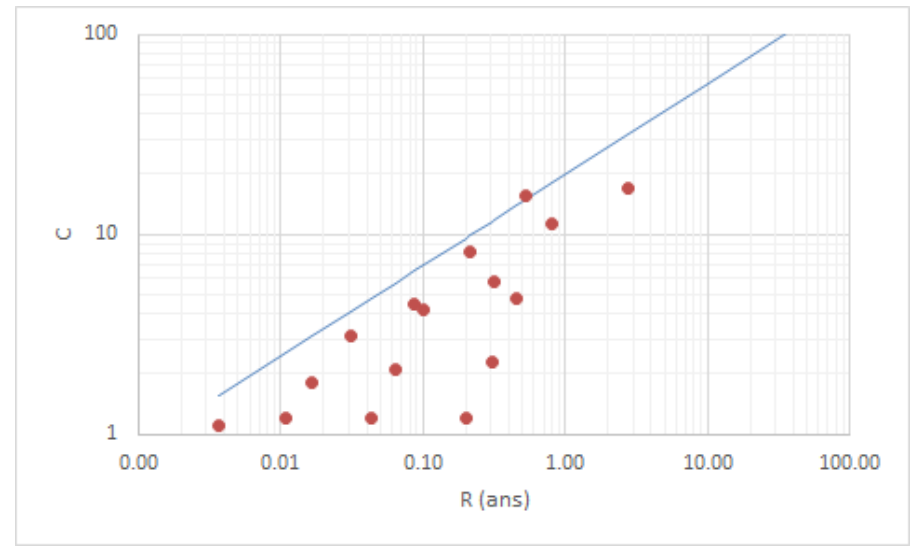

Figure 3. Coefficient de corrélation Hmo-Zw.

Sur cette base, on en déduit une variation de $C$ en puissance de $N R$, qui se traduit par la courbe bleue, soit un coefficient de corrélation $C_{100}$ voisin de 160 . Ceci se traduit par le diagramme qui donne les couples de durées de retour $R_{H s}$ et $R_{Z w}$ associés à une durée de retour globale donnée (figure 4).

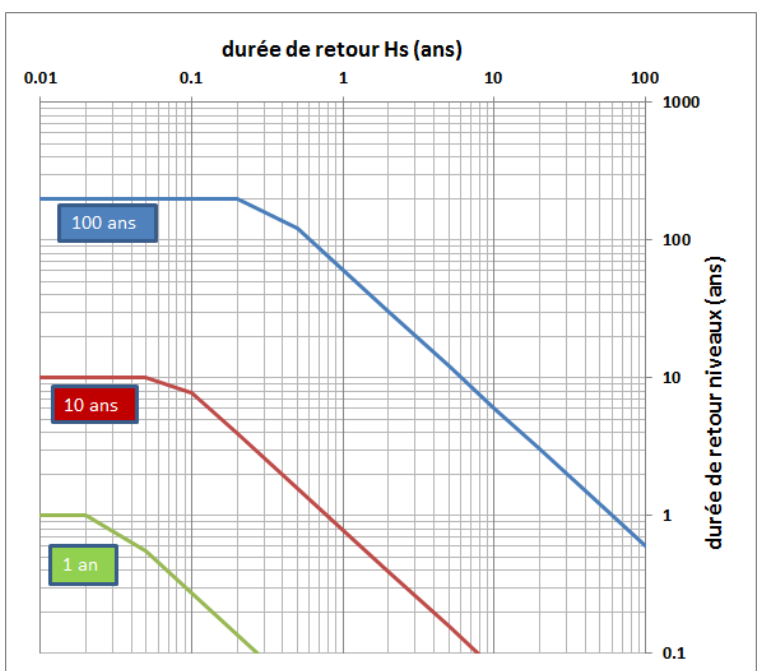

Figure 4. Diagrammes de corrélation entre la période de retour de Hs et Zw. 


\section{XVèmes Journées Nationales Génie Côtier - Génie Civil \\ La Rochelle, 29 au 31 mai 2018}

\subsection{Environnement}

Le projet s'insère dans une zone sensible à plusieurs titres :

- proximité immédiate des deux réserves marines de Monaco : à l'ouest la réserve des Spélugues et son tombant coralligène associant des espèces remarquables et protégées, telles le corail rouge; à l'est, la réserve du Larvotto et son herbier de posidonies, écosystème remarquable et protégé en Méditerranée,

- présence dans l'emprise d'espèces protégées (posidonie, grande nacre, mérou),

- problématique de dragage et de maîtrise de la turbidité,

- proximité immédiate d'hôtels et d'habitations particulières de très haut standing, et d'un centre d'expositions et de congrès (le Grimaldi Forum), dont un des objectifs du projet est d'en assurer l'extension,

- proximité de Port Hercule, dont on ne doit pas augmenter l'agitation, ce qui suppose de minimiser le coefficient de réflexion des ouvrages, et prise en compte du risque de choc accidentel de bateaux,

- proximité des plages du Larvotto, principal espace balnéaire de la Principauté.

Ces aspects ont été pris en compte dans la conception même du projet et dans la mise au point des méthodes de travaux.

\section{Le concept d'infrastructure}

\subsection{Principe des caissons}

Après analyse multicritères de l'ensemble des solutions techniques possibles, le concept d'une ceinture de caissons fondés sur des assises en matériaux grossiers, avec des bâtiments fondés sur pieux a été retenu. Un remblai de coefficient de frottement élevé est prévu dans la zone du coin de poussée des caissons, les immeubles étant sauf exception fondés sur pieux comme représenté sur la coupe schématique de la figure 5.

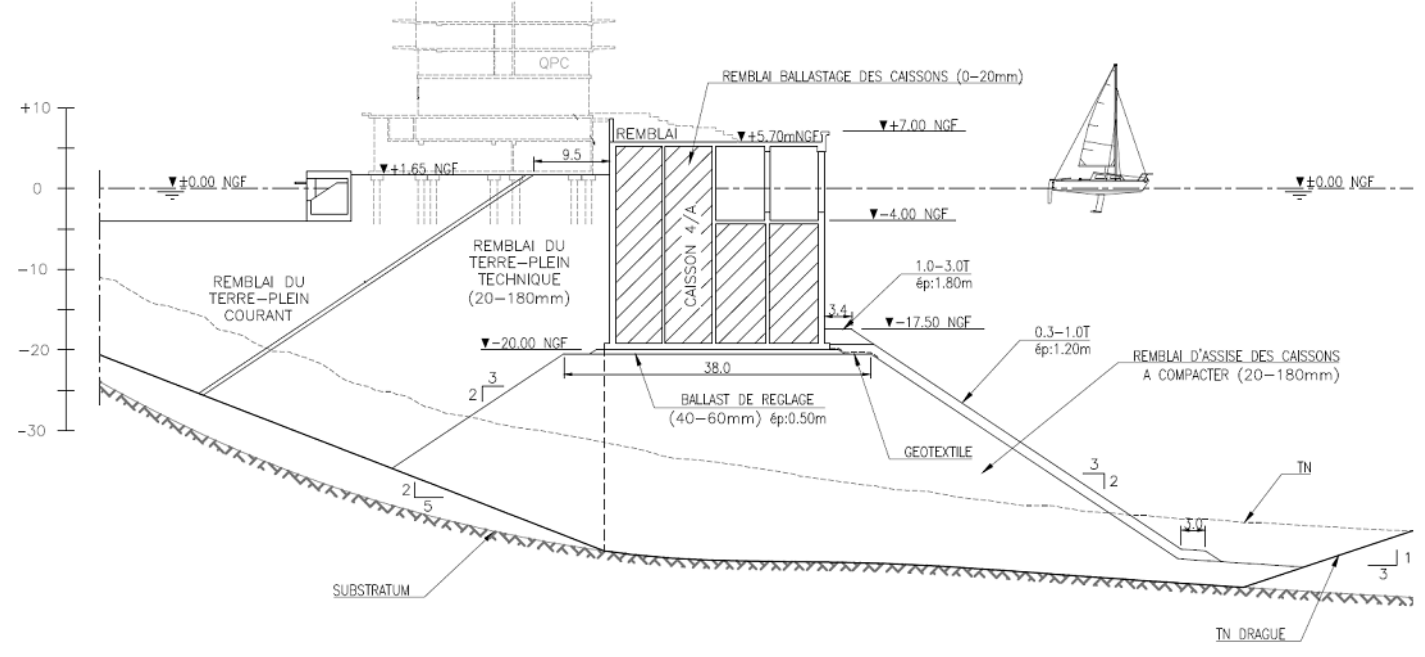

Figure 5. Coupe-type caisson et immeuble. 


\section{Thème 4 - Ouvrages portuaires, offshore et de plaisance}

Le projet peut être divisé en trois zones principales: le port d'animation et la promenade Fairmont, la ceinture principale de caissons (du caisson-piscine $\mathrm{C} 0$ au caisson C17), la zone du Larvotto (figure 6). Afin d'épouser au mieux les formes du plan-masse, des caissons de forme trapézoïdale ont été imaginées, afin de constituer les briques élémentaires de la ceinture.

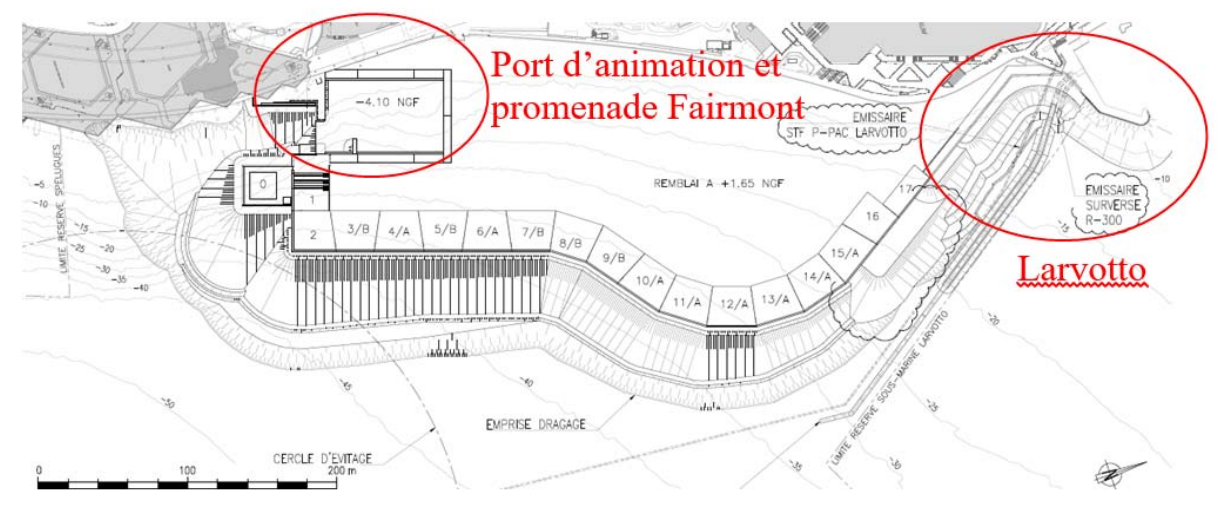

Figure 6. Forme et disposition des caissons.

\subsection{Conception hydraulique des caissons}

Des essais en canal à houle (échelle 1/40) ont été effectués chez Océanide, tant pour optimiser le coefficient de réflexion que pour mesurer les franchissements et les efforts sur les caissons et vérifier la stabilité de la protection de l'assise (figure 7).

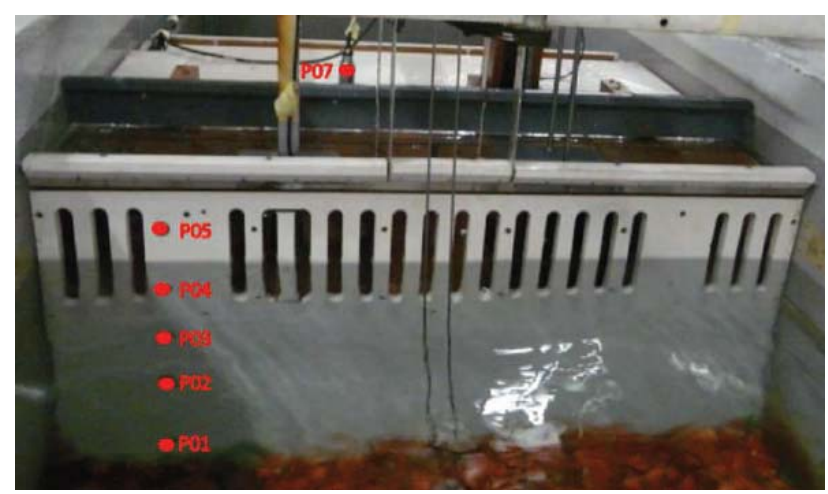

Figure 7. Maquette du caisson avec capteurs de pressions.

Les franchissements sont censés être limités à $0.1 \mathrm{1} / \mathrm{m} / \mathrm{s}$ sous houle décennale sur la promenade extérieure le long des caissons. Au-delà de cette limite de houle décennale, la promenade peut être fermée au public pour éviter tout risque pour les piétons, et la maîtrise des franchissements concerne dans ce cas la protection des immeubles situés à l'arrière des caissons. Une première optimisation des chambres et des parapets a été effectuée en canal à houle sur la base de ce critère décennal. 


\section{XVèmes Journées Nationales Génie Côtier - Génie Civil \\ La Rochelle, 29 au 31 mai 2018}

Les essais en canal ont également été mis à profit pour mesurer le coefficient de réflexion global du caisson sur son assise, dans l'objectif de minimiser les réflexions parasites vers Port Hercule. Les coefficients de réflexion obtenus ont été de l'ordre de 0.35 , ce qui est très faible pour une paroi de cette hauteur.

Compte-tenu des forts effets 3D, des essais en cuve (échelle 1/60) ont été effectués chez HR Wallingford pour valider le concept hydraulique final d'infrastructure, visant à évaluer la stabilité des protections (assises des caissons, des quais-blocs de la zone Larvotto et de la promenade Fairmont) et les franchissements (figure 8).
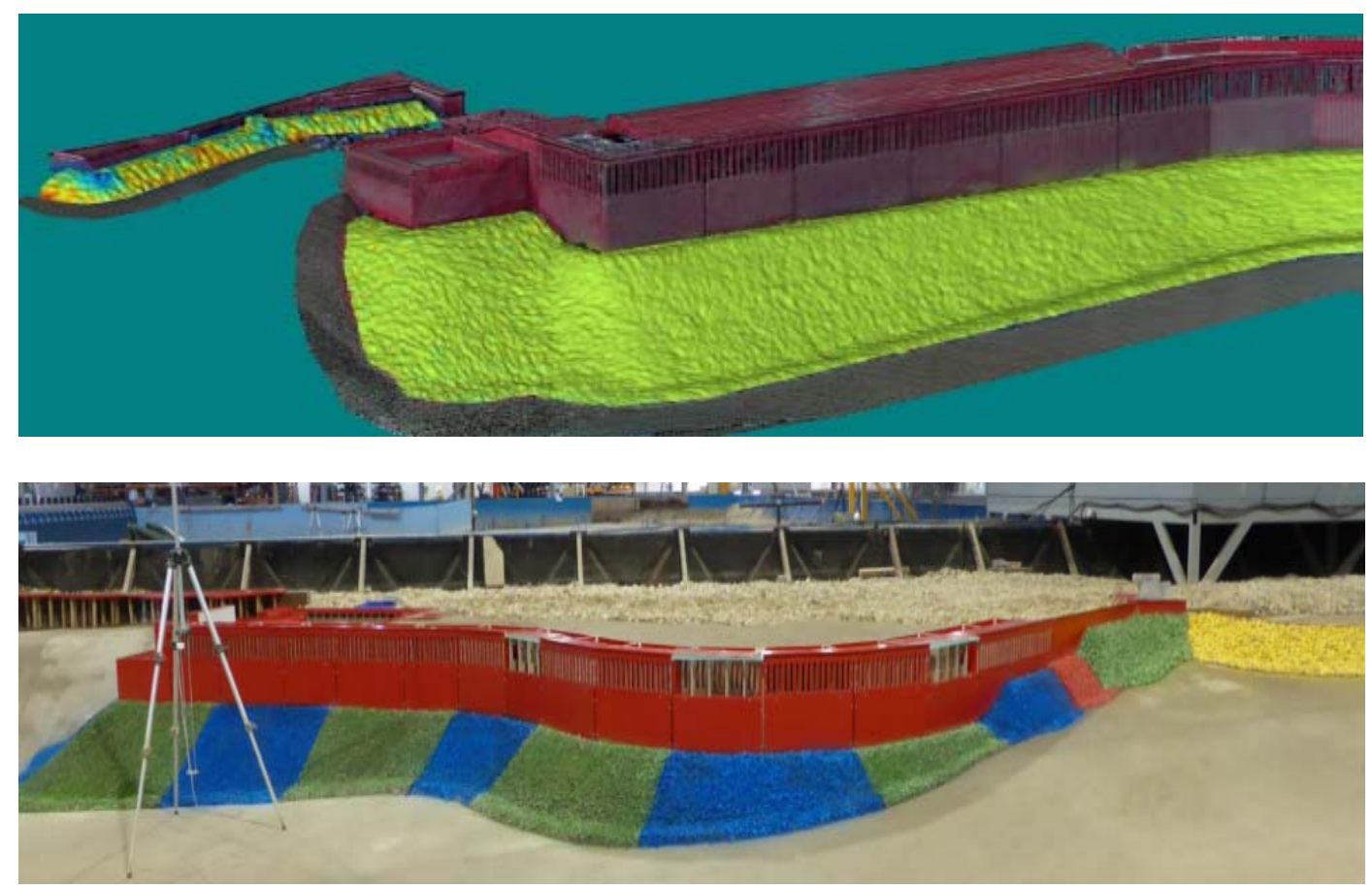

Figure 8. Vue d'ensemble du modèle en cuve et relevé laser d'érosion d'assises.

\subsection{Stabilité et aspects structurels}

Du fait que les caissons sont adossés à un remblai sur toute leur longueur, la houle n'est susceptible d'impacter la stabilité des caissons que lors du creux de vague, qui se superpose à la poussée statique du remblai. Mais c'est en définitive le séisme qui s'est avéré le cas dimensionnant : le séisme de non-effondrement est de $0.16 \mathrm{~g}$ au substratum, pondéré par un coefficient d'importance de 1.2, lui-même amplifié par l'épaisseur de sol meuble d'un facteur 1.5 , ce qui conduit à une accélération de calcul de $0.28 g$ sur un soutènement de plus de $20 \mathrm{~m}$ de hauteur.

La poussée sismique sur les caissons, évaluée par la méthode de Mononobe-Okabe, a été combinée quadratiquement avec les efforts sismiques transmis par les pieux supportant les immeubles, ceux-ci ayant été calculés par Tractebel via un modèle dynamique bidimensionnel intégrant les fondations des bâtiments, le remblai, les 


\section{Thème 4 - Ouvrages portuaires, offshore et de plaisance}

caissons et leur assise, et après avoir vérifié que les fréquences des immeubles et des caissons étaient bien distinctes.

La condition sismique s'est révélée de manière générale comme l'action dimensionnante vis-à-vis de la stabilité locale (glissement et poinçonnement) et de la stabilité d'ensemble, ce qui a conduit à spécifier des matériaux de fort coefficient de frottement pour l'assise et le coin de poussée, et à draguer la totalité des sédiments sous l'emprise des caissons).

Les essais en canal ont également fourni des pressions, qui ont été traduites en diagrammes de pression à appliquer au calcul structurel.

\section{La zone du port d'animation}

\subsection{L'agitation dans le port d'animation}

Les quais devaient respecter, pour des considérations d'urbanisme, un niveau imposé relativement bas à $+2.15 \mathrm{~m}$ NGF.

La coupe-type de la figure 9 a été retenue, afin de préserver un coefficient de réflexion aussi bas que possible, en en s'imposant un ponton fixe à $+0.80 \mathrm{~m}$ NGF, passant à $+1.20 \mathrm{~m}$ NGF à l'horizon 2100. Les fentes de dissipation sont d'une porosité de $50 \%$.
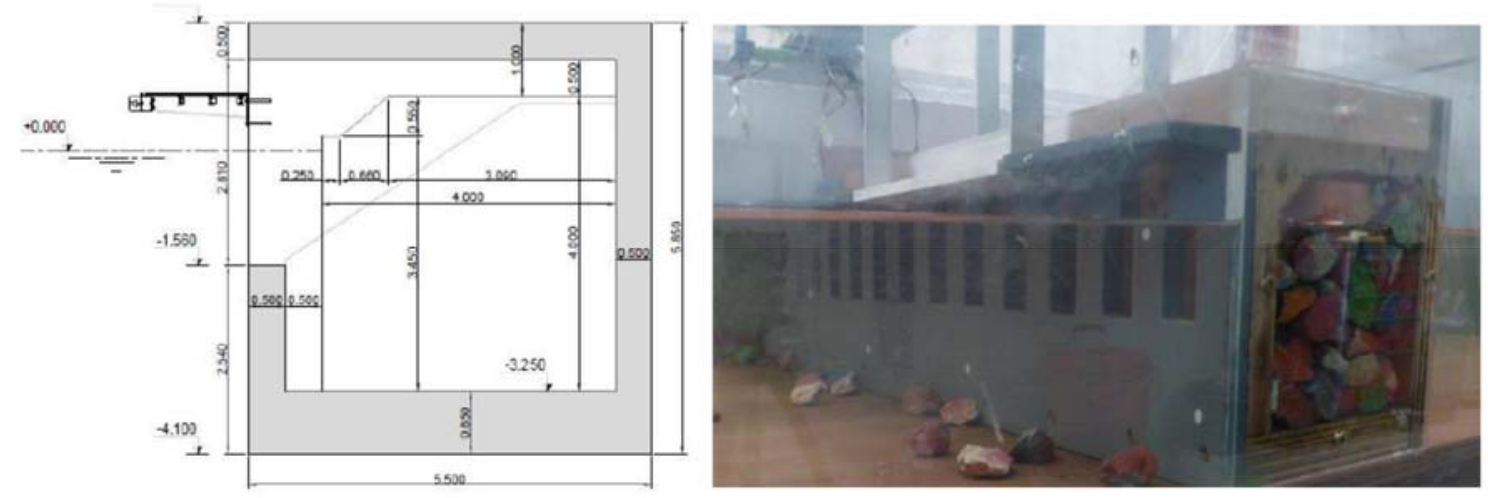

Figure 9. Coupe-type et photo de la maquette 2D du quai du port d'animation.

Des essais en canal au 1/30 ont été effectués chez Océanide, tant pour optimiser le coefficient de réflexion que pour mesurer les efforts sur la structure en béton armé et vérifier la stabilité des enrochements.

Les coefficients de réflexion $C_{r}$ suivants ont été obtenus pour des $H_{m o}$ incidents de l'ordre de $0.3 \mathrm{~m}$ ( $C_{r}$ diminuant légèrement quand on passait de $H_{m o}=0.3$ à $\left.0.5 \mathrm{~m}\right)$. On note l'influence du niveau d'eau $Z_{w}, C_{r}$ augmentant avec le niveau d'eau du fait que la chambre se retrouve de plus en plus saturée, ainsi que par l'effet défavorable du relèvement du ponton à l'horizon 2100 (figure 10). 


\section{XVèmes Journées Nationales Génie Côtier - Génie Civil \\ La Rochelle, 29 au 31 mai 2018}

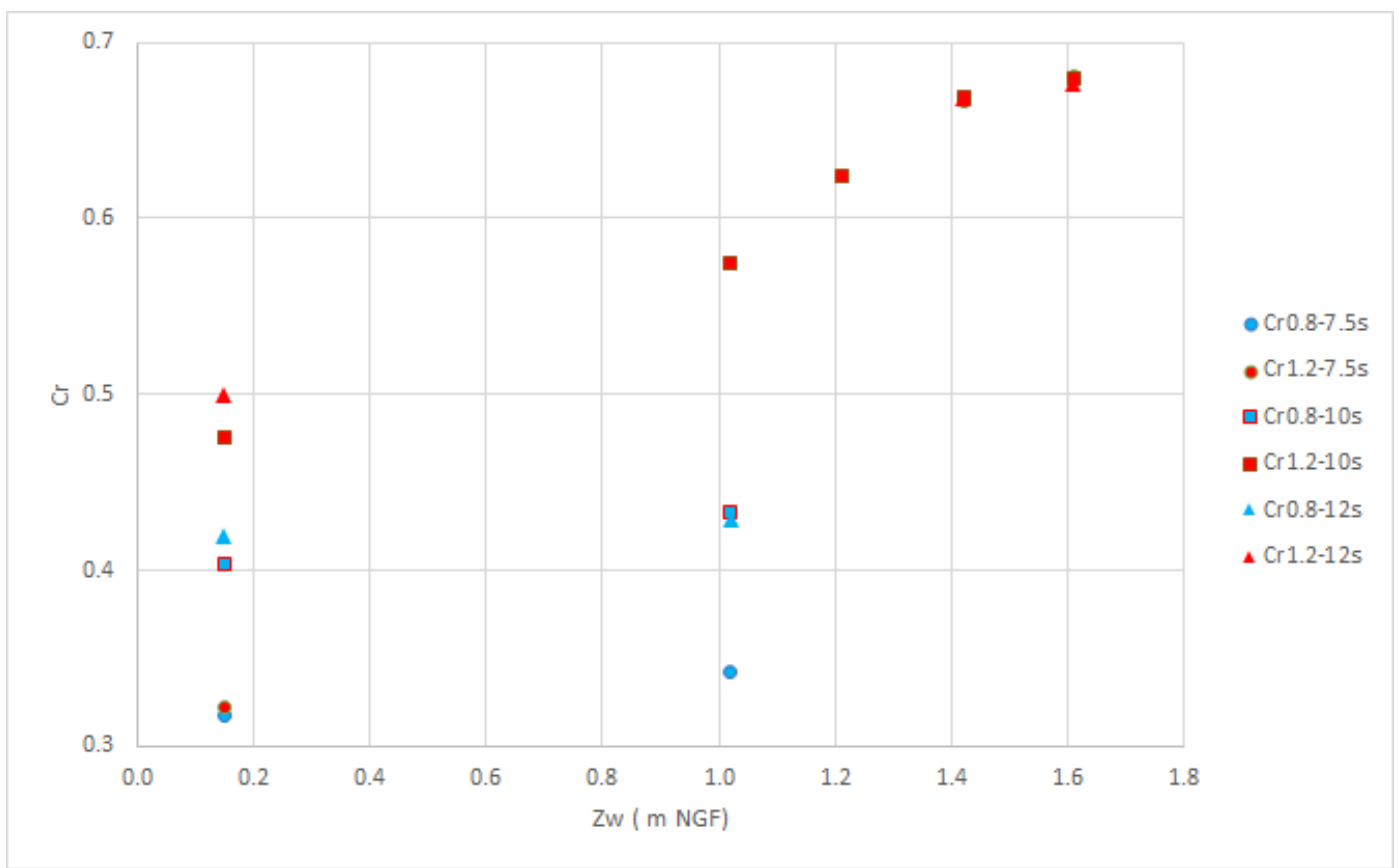

Figure 10. Coefficients de réflexion du quai.

L'agitation dans le port a été évaluée sur modèle numérique (code Refonde du Cerema, figure 11) sur la base de valeurs conservatives de ces coefficients (0.6 à l'horizon 2060 et 0.8 en 2100). Plus précisément, un balayage en direction et période a été effectué pour des houles de hauteur unité à l'entrée du modèle, ce qui a permis d'obtenir des matrices de transfert $K_{d}\left(\theta, T_{p}\right)$ pour chaque zone du port. Ces matrices ont permis de reconstituer des statistiques d'agitation dans chaque zone sur la base du fichier temporel d'états de mer déduit du hindcasting évoqué plus haut.

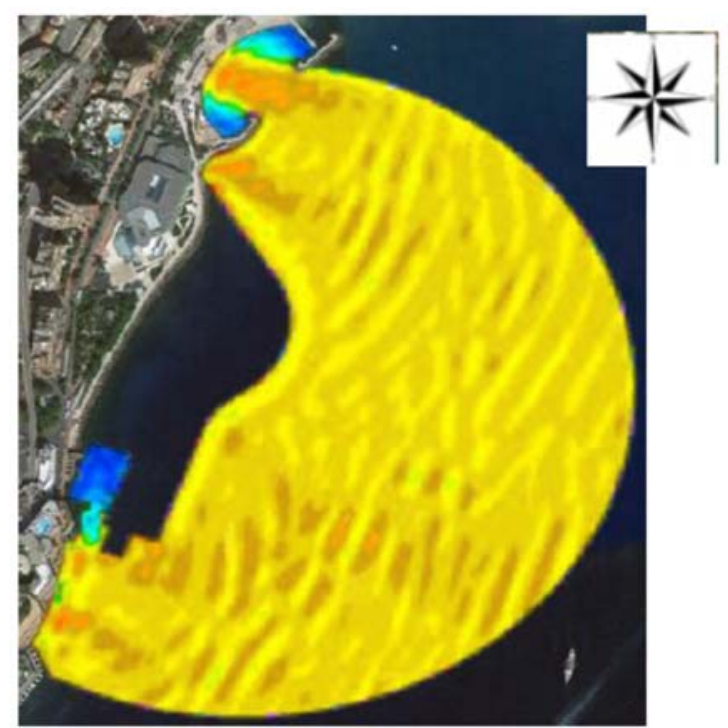

Figure 11. Carte d’iso-agitation du modèle numérique Refonde. 


\section{Thème 4 - Ouvrages portuaires, offshore et de plaisance}

On a également construit un second modèle numérique englobant Port Hercule et sa digue transmissible, en considérant cette fois pour les houles de secteur sud non plus le point $\mathrm{Pt} 3$ (qui incluait la digue transmissible) mais le point Pt5. Les résultats ont été très similaires, validant le principe d'avoir intégré la digue dans le hindcasting.

Bien que le modèle physique 3D réalisé à $\mathrm{HR}$ Wallingford n'ait pas eu pour but de mesurer l'agitation (problème d'échelle pour des hauteurs dans le port de l'ordre de $30 \mathrm{~cm}$ ), on a confronté le modèle numérique avec les agitations mesurées en cuve sous conditions centennales de Nord-Est : les hauteurs mesurées se sont révélées tout à fait cohérentes avec les iso-valeurs issues de la modélisation numérique (figure 12) :

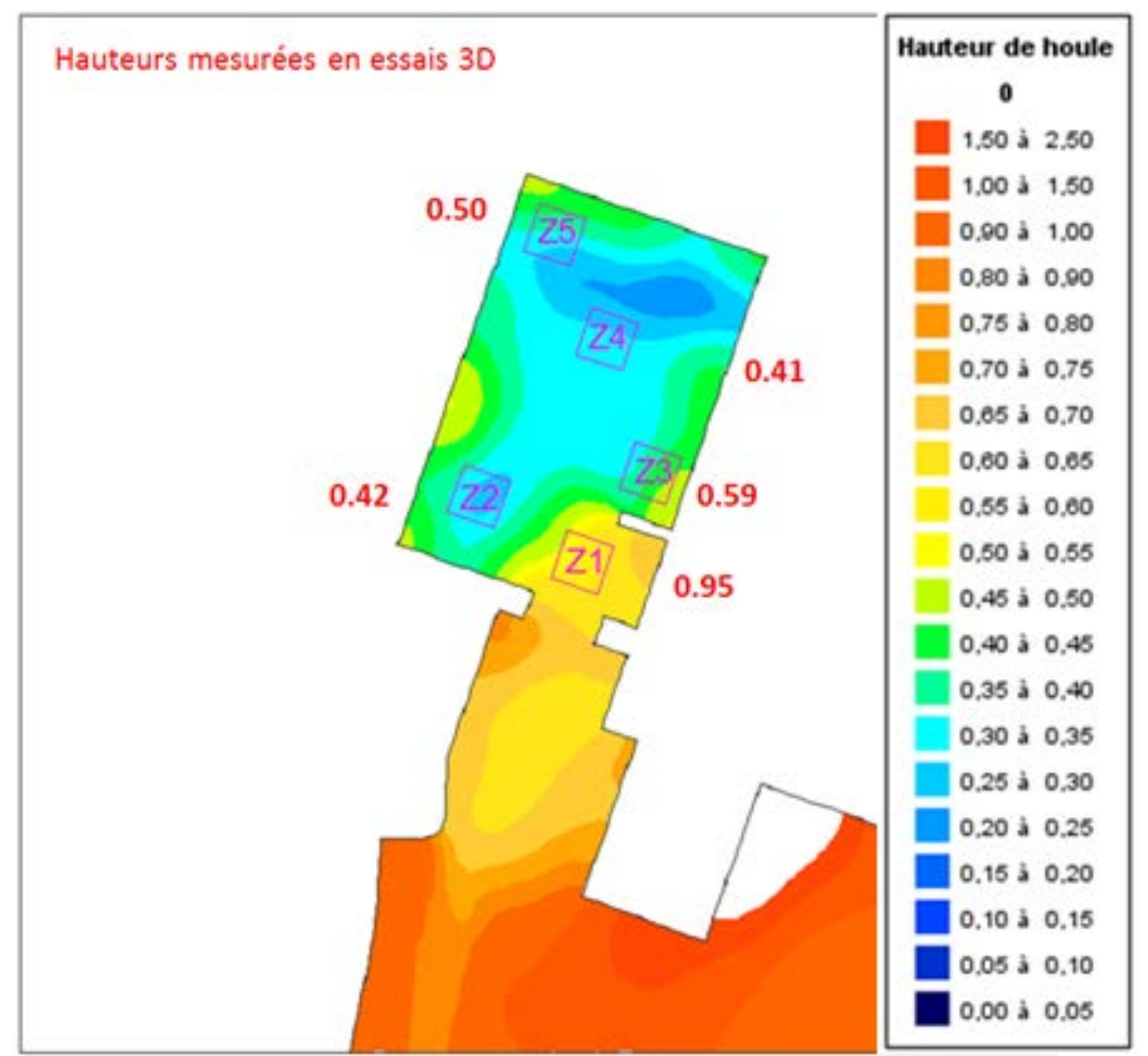

Figure 12. Comparaison entre isohauteurs calculées et mesures ponctuelles.

4.2 Les franchissements dans la zone du port d'animation et de la promenade Fairmont Un important travail d'optimisation a été mené, en liaison étroite avec les architectes et paysagistes, pour gérer les franchissements dans cette zone tout en cherchant à minimiser au maximum les hauteurs de parapets et à éviter les barrières aux flux de piétons. 


\section{XVèmes Journées Nationales Génie Côtier - Génie Civil \\ La Rochelle, 29 au 31 mai 2018}

De nombreuses configurations de tenons, parapets, épis, systèmes de drainage, ont ainsi été testées en cuve chez HR Wallingford (figure 13).

Les phénomènes étaient très largement tridimensionnels, et les structures trop atypiques pour entrer dans une quelconque catégorie d'ouvrage pour laquelle on disposerait de formules empiriques (par exemple un escalier au niveau de l'eau en tête de talus).
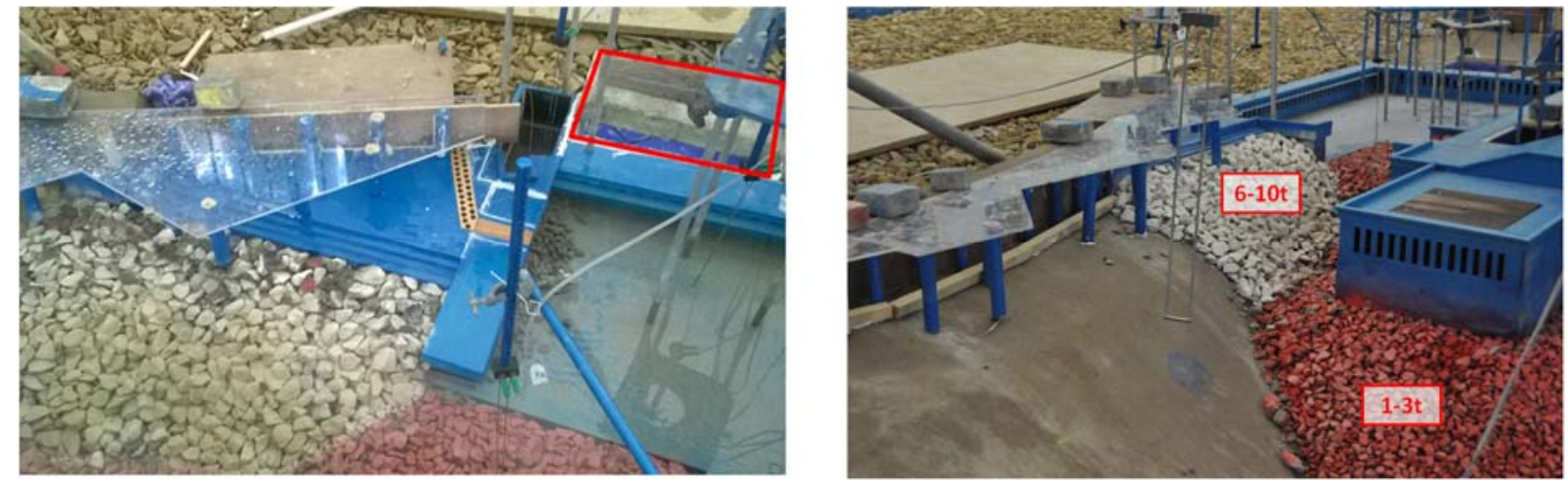

Figure 13. Modélisation physique de l'avant-port et de la promenade Fairmont.

\subsection{Le caisson-piscine}

Sauf à réaliser des tenons et/ou des murets anti-franchissement qui ne serviraient que sous forte tempête et bloqueraient la vue le reste du temps, et dès lors que le principe de protections amovibles avait été écarté par le Maître d'Ouvrage, on a dû adjoindre au projet une digue.

Cette "digue" s'est rapidement transformée en "caisson-piscine", que l'on peut voir à droite sur la figure précédente ou en perspective ci-dessous. Les fentes qui apparaissent sur la figure ci-dessous sont des fenêtres en plexiglas, l'idée étant d'obtenir un aspect aussi homogène que possible avec le reste des parements dotés de fentes de dissipation des houles (figure 14).

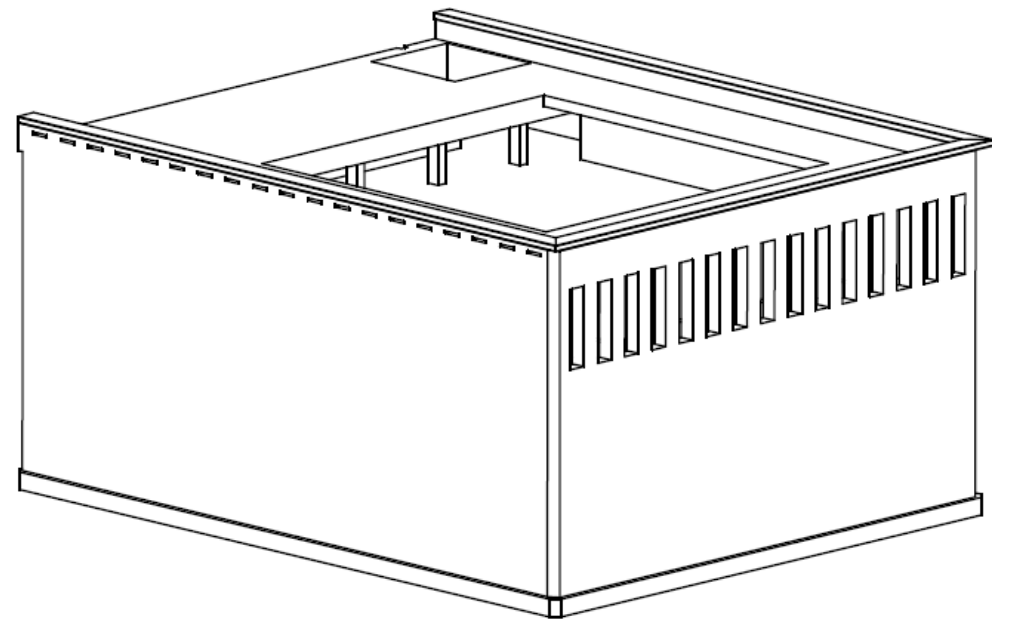

Figure 14. Vue 3D du caisson-piscine. 


\section{Thème 4 - Ouvrages portuaires, offshore et de plaisance}

Une évaluation assez fine des impacts de houle sur chaque partie de structure a dû être effectuée pour les dimensionner, y compris des points singuliers comme le fond mobile de la piscine ou les vitres en plexiglas aménagées dans la paroi exposée côté Est.

\section{La zone du Larvotto}

L'enjeu dans cette zone était d'éviter un impact de la réflexion des houles sur les derniers caissons sur les épis de protection des plages, dont le dimensionnement a été effectué il y a plusieurs décennies et ne prenait bien évidemment pas en compte le projet de l'Anse du Portier, ni les houles de dimensionnement définies pour celui-ci.

Il s'agissait également d'éviter des concentrations d'agitation à la jonction entre le nouveau plan masse et le tracé de la côte existante, qui crée à cet endroit une singularité propice à la concentration de l'énergie de la houle.

Comme pour les autres sujets évoqués précédemment, une combinaison d'approches numériques et d'essais en cuve à houle ont permis d'écarter tout risque.

\section{Conclusion}

Le projet de l'Anse du Portier est un projet exceptionnel par son ampleur et son contexte (conditions de site sévères, zones à fort enjeu environnemental, contraintes antagonistes, ouvrages de type portuaire à fonction urbaine, ...).

La conception de l'infrastructure n'a pu se faire qu'en étroite collaboration avec toutes les parties prenantes (tant au niveau du concept d'ensemble que dans les "détails" qui ont occasionné de longues itérations.

Il a également été l'occasion de réaliser des actions environnementales originales, parmi lesquelles la transplantation de posidonies et l'accrochage en diverses parties des caissons (chambre de dissipation et parement) d'habitats artificiels destinés à favoriser la présence d'espèces de faune et flore marines fixées et vagiles.

Les raccordements à l'existant ont été également des ouvrages à part entière, car leur conception a dû jouer avec des contraintes d'espace drastiques (tant côté Fairmont que côté Larvotto), de recherche d'homogénéité visuelle, ou encore avec la stabilité d'ouvrages existants dimensionnés sur la base d'hypothèses très différentes du projet. 